\title{
Cloud based mobile solution for early detection of Skin Cancer using Artificial Intelligence
}

\author{
Pawan Sonawane, Sahel Shardhul , Raju Mendhe \\ Computer Engineering, Datta Meghe College of Engineering, Airoli, Navi Mumbai, Maharashtra, India
}

\begin{abstract}
Article Info

Volume 7, Issue 3

Page Number: 312-324

Publication Issue :

May-June-2021

\section{Article History}

Accepted : 15 May 2021

Published : 22 May 2021

The vast majority of skin cancer deaths are from melanoma, with about 1.04 million cases annually. Early detection of the same can be immensely helpful in order to try to cure it. But most of the diagnosis procedures are either extremely expensive or not available to a vast majority, as these centers are concentrated in urban regions only. Thus, there is a need for an application that can perform a quick, efficient, and low-cost diagnosis. Our solution proposes to build a server less mobile application on the AWS cloud that takes the images of potential skin tumors and classifies it as either Malignant or Benign. The classification would be carried out using a trained Convolution Neural Network model and Transfer learning (Inception v3). Several experiments will be performed based on Morphology and Color of the tumor to identify ideal parameters.

Keywords : Machine Learning, Artificial Intelligence, Deep Learning, Cloud Computing.
\end{abstract}

\section{INTRODUCTION}

Skin cancer is a dangerous and widespread disease. Each year there are approximately 5.4 million new cases of skin cancer are recorded in USA alone .The global statistics are equally alarming. Recent reports show that from 2008 to 2018, there has been a 53\% increase in new melanoma cases diagnosed annually. The mortality rate of this disease is expected to rise in the next decade. The survival rate is less than $14 \%$ if diagnosed in later stages. However, if the skin cancer is detected at early stages then the survival rate is nearly $97 \%$. This demands the early detection of skin cancer.

Skin cancer may appear as malignant or benign form. Benign Melanoma is simply appearance of moles on skin. Malignant melanoma is the appearance of sores that cause bleeding. Malignant Melanoma is the deadliest form of all skin cancers. It arises from cancerous growth in pigmented skin lesion. The vast majority of skin cancer deaths are from melanoma, with about 1.04 million cases annually. Melanoma diagnosis is difficult and needs sampling and laboratory tests. It can spread out to all parts of the body through lymphatic system or blood. If diagnosed at the right time, this disease is curable.

The main problem to be considered dealing with melanoma is that, the first affliction of the disease can pave the way for future ones. Laboratory sampling often causes the inflammation or even spread of lesion. Thus, there is a need for an 
application that can perform a quick, efficient, and low-cost diagnosis. Computer based diagnosis can improve the speed of skin cancer diagnosis which works according to the disease symptoms.

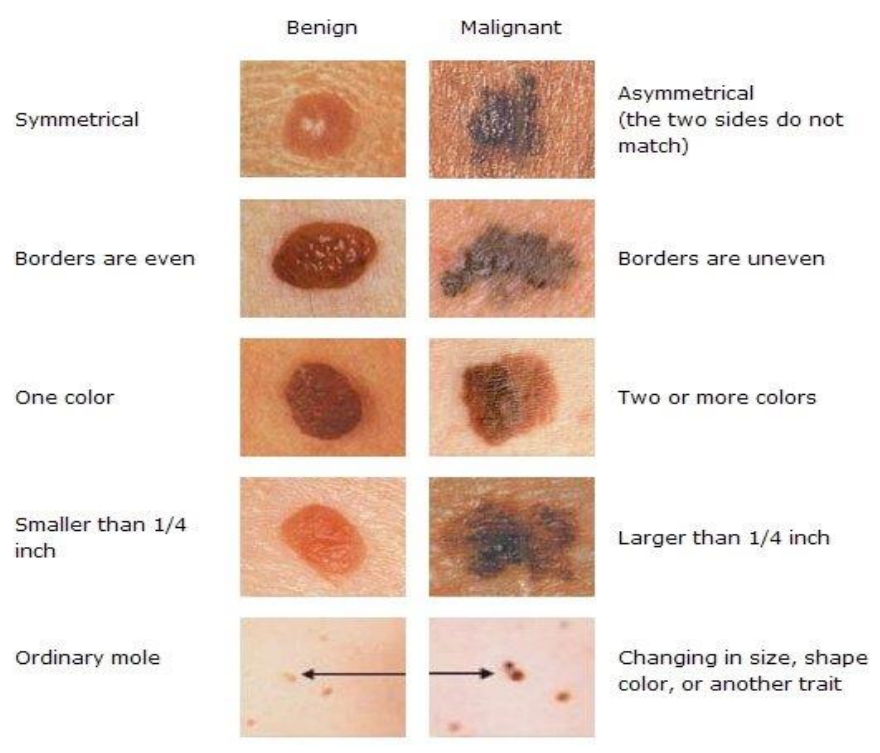

Fig 1.1 Benign vs Malignant Melanoma

Our solution proposes to build a serverless mobile application on the AWS cloud that takes the images of potential skin tumors and classifies it as either Malignant or Benign. The classification would be carried out using a trained Convolution Neural Network model and Transfer learning (Inception v3). Several experiments will be performed based on Morphology and Color of the tumor to identify ideal parameters.

Skin Cancer is the cancer affecting the skin. Skin cancer may appear as malignant or benign form. Benign Melanoma is simply appearance of moles on skin. Malignant melanoma is the appearance of sores that cause bleeding. Malignant Melanoma is the deadliest form of all skin cancers.

It arises from cancerous growth in pigmented skin lesion. Malignant melanoma is named after the cell from which it presumably arises, the melanocyte. If diagnosed at the right time, this disease is curable.
Melanoma diagnosis is difficult and needs sampling and laboratory tests. Melanoma can spread out to all parts of the body through lymphatic system or blood. The main problem to be considered dealing with melanoma is that, the first affliction of the disease can pave the way for future ones.

Laboratory sampling often causes the inflammation or even spread of lesion. So, there has always been lack of less dangerous and time-consuming methods. Computer based diagnosis can improve the speed of skin cancer diagnosis which works according to the disease symptoms.

The similarities among skin lesions make the diagnosis of malignant cells a difficult task. But there are some unique symptoms of skin cancer, such as: Asymmetry, Border irregularity, Color variation and Diameter. Those are popularly known as $A B C D$ parameters. $\mathrm{ABCD}$ parameters. Asymmetry, Border irregularity, Color, Diameter. Asymmetry is one half of the tumor does not match the other half. Border Irregularity is the unevenness of images. Color intensity change in the lesioned region is irregular. Malignant melanoma is having a diameter greater than $6 \mathrm{~mm}$.

\section{LITERATURE REVIEW}

\subsection{Papers and Literature Review}

There is a rising need for automatic skin cancer detection system with high accuracy because the early detection of this disease can be very crucial and life-saving. There are two types of image available for skin cancer detection. The dermoscopy image is captured by specialized dedicated system in pathological center with focused on region of interest with high zoom (E.g. 20x), which needs skilled dermatologist to conclude the image as positive or negative. 
This type of image can be feed to computerized semiautomated system for classification. But in this technology, the victim always needs to walk-into the pathological center and need to take consultancy of the skilled dermatologist. On the other hand, if there is a computer software which can automatically detect skin cancer from digital image captured by any digital image capturing system with little focus on the region of interest, victim can anytime perform the test even at home.

Hence the motivation of this literature survey is to understand the recent technology for skin cancer cell detection and focus on developing an automatic system for skin cancer detection from digital image using deep learning technology.

1. Rahil Garnavi proposed an optimized selection and integration of features derived from textural, border based, and geometrical properties of the melanoma lesion. The texture features were derived from using wavelet-decomposition, the border features were derived from constructing a boundary-series model of the lesion border and analyzing it in spatial and frequency domains, and the geometry features were derived from shape indexes. Classification was done through the use of four classifiers; namely, support vector machine, random forest, logistic model tree, and hidden naive Bayes.

2. Farzam Kharaji Nezhadian and Saeid Rashidi proposed new algorithm presented to classify dermoscopic images into malignant and benign. Initially the images were segmented using active counter model and two features such as texture and colorful components were extracted. Texture-based features were first in this area used to diagnose disease and its results indicated high-efficacy. In the international skin imaging collaboration dataset, they achieved accuracy of 97\% by support vector machine classifier.

3. Ms. H. R. Mhaske and Mrs. D. A. Phalke presented a paper with both supervised and unsupervised classification, done using supervised learning-based classifiers as Neural Network, Support Vector Machine and unsupervised learning-based classification as K-means clustering algorithm. The resultant accuracy was compared with these different classifiers. The highest accuracy was obtained by Support Vector Machine.

4. Xiangfeng Dai, Irena Spasić, Bradley Meyer, Samuel Chapman and Frederic Andres presented an on-device inference App and used a dataset of skin cancer images to demonstrate a proof of concept to tackle the challenges of mHealth applications. They pre-trained a Convolutional Neural Network model using 10,015 skin cancer images. The model was then deployed on a mobile device, where the inference process takes place, i.e. when presented with new test image all computations were executed locally where the test data remained. This approach reduced latency, saved bandwidth and improved privacy.

5. M. Emre Celebi, Hassan A. Kingravi, Bakhtiyar Uddin, Hitoshi Iyatomi, Y. Alp Aslandogan, William V. Stoecker, and Randy H. Moss in this paper presented a methodological approach to the classification of pigmented skin lesions in dermoscopy images. First, automatic border detection was performed to separate the lesion from the background skin. Shape features were then extracted from this border. For the extraction of color and texture related features, the image was divided into various clinically significant regions using the Euclidean distance transform. This feature data was fed into an optimization framework, which ranks the features using various feature selection algorithms and determines the optimal feature subset size according to the area 
under the ROC curve measure obtained from support vector machine classification.

6. Esperanza Guerra-Rosas and Josué ÁlvarezBorrego proposed methodology based on Fourier spectral analysis by using filters such as the classic, inverse and k-law nonlinear. The sample images were obtained by a medical specialist and a new spectral technique was developed to obtain a quantitative measurement of the complex pattern found in cancerous skin spots. Finally, a spectral index was calculated to obtain a range of spectral indices defined for skin cancer. Their results showed a confidence level of $95.4 \%$.

7. Ilias Maglogiannis and Charalampos N. Doukas, in their paper, reviewed the state of the art in such systems by first presenting the installation, the visual features used for skin lesion classification, and the methods for defining them. Then, they described how to extract these features through digital image processing methods, i.e., segmentation, border detection, and color and texture processing, and they presented the most prominent techniques for skin lesion classification.

8. Vincent T.Y. Ng, Benny Y.M. Fung and Tim K. Lee proposed an adaptive fuzzy approach that used symmetric distance (SD) to measure lesions with fuzzy borders. The use of a number of SD variations and the adoption of a back propagation neural network enhanced the discriminative power of the approach. Digitized images from the Lesion Clinic in Vancouver, Canada, demonstrated the accurate classification of asymmetric lesions at around $80 \%$.

9. Vijayalakshmi $M$ presented a completely automated system of dermatological disease recognition through lesion images, a machine intervention in contrast to conventional medical personnel-based detection. Her model was designed into three phases compromising of data collection and augmentation, designing model and finally prediction. She had used multiple AI algorithms like Convolutional Neural Network and Support Vector Machine and amalgamated it with image processing tools to form a better structure, leading to higher accuracy of $85 \%$.

\subsection{Summarized Findings}

- Buka: There's one technology called MelaFind that uses infrared light to evaluate pigmented lesions up to a few millimeters below the skin. Then sophisticated AI algorithms evaluate that scanned data to diagnose serious skin cancers, including melanoma.

- While the technology is promising, the margin of error isn't yet small enough to warrant using it in lieu of a biopsy, for example. That being said, there are patients around the world without access to quality dermatological care. For those patients, this kind of AI can already help save lives, and it's getting better all the time.

- ANN: An artificial neuron network (ANN) is a statistical nonlinear predictive modeling method which is used to learn the complex relationships between input and output. ANN learns weight of each connecting link by forward and backward propagation. For skin cancer detection the total number of nodes or layers in each ANN is dependent on the number of input images used.

- Back propagation is a method used in artificial neural networks to calculate the error contribution of each neuron after a batch of data (in image recognition, multiple images) is processed. This is used by an enveloping optimization algorithm to adjust the weight of each neuron, completing the learning process for that case. Technically, it calculates the gradient of the loss function. It is commonly used in the gradient descent optimization algorithm. It is also called backward propagation of errors, because the error is calculated at the output and distributed back through the network layers. 
- Support vector Machine is a supervised nonlinear classifier which constructs an optimal n-dimensional hyperplane to separate all the data points in two categories. SVM can be used for classification of skin lesions such as Basal cell carcinoma (BCC), Melanoma, Seborrheic keratosis (SK). Some techniques use SVM as multi-class detection algorithm. SVM is used to detect four different class - Melanocytic Skin Lesion (Melanoma and Nevus), Non-Melanocytic Skin Lesion (BCC and SK).

\section{REQUIREMENT ANALYSIS}

\subsection{Software Requirements}

i. Android OS 4.4 and above supported devices to host the mobile application which will run the classification of images captured of the affected skin area on the cloud system.

ii. Python3 - Extensive libraries in Python like Scipy, matplotlib etc. provides a lot of help and ease the system development.

iii. Cloud Services - Amazon Web Services (AWS) is used for scalability, storage and infrastructure requirements.

\subsection{Hardware Requirements}

- Mobile camera - mobile camera will be used to capture images of the affected skin area in order to store and transmit the skin images on the mobile device and cloud.

- Internet Connectivity - Good internet connectivity is essential for smooth operation of the application. The heart sound after being uploaded on the mobile device needs to be classified using cloud-based system which requires internet connection to communicate with the cloud system.

\section{Project Design}

\subsection{Architecture}

Architecture illustrates the interaction between the various components of the system. The system primarily consists of a mobile app and a cloud platform. The cloud platform further comprises Image pre-processor, CNN classifier and trainer, training database and post-processor.

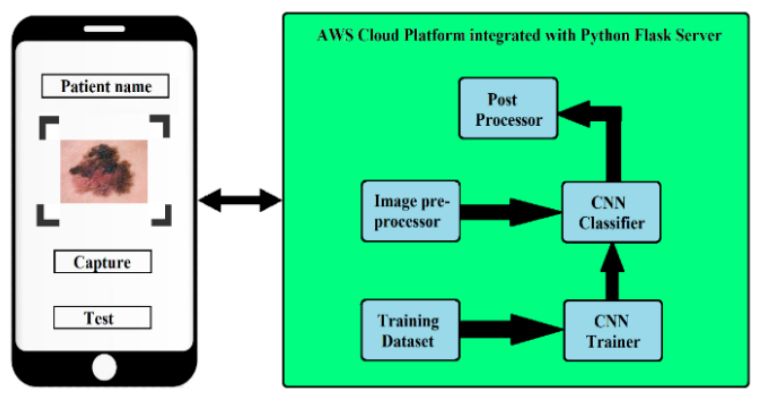

Fig 4.1.1 System Architecture

According to Fig 4.1.1, the system comprises 6 main components:

4.1.1 Mobile Application - Will contain a simplified frontend for the users to enter their information and capture skin images by interfacing with the mobile camera. Save the skin images as either .jpg or .png. Upload it to the Cloud platform for analysis and return the diagnosis to the user whether the image is Malignant or Benign. Provides additional functionality to email the results to a doctor.

4.1.2 Image pre-processor - Cloud-based Python program to standardize the images, perform median filtering, segmentation and feature extraction on the image before passing it to the $\mathrm{CNN}$ classifier.

4.1.3 CNN Trainer - Cloud-based program that trains a CNN model (Inception v3) to classify images. CNN model will be trained with benign and malignant tumor images from reliable open source repositories. 
4.1.4 CNN Classifier - Cloud-based program that categorizes input images as either benign or malignant using the trained model from the CNN Trainer.

\subsubsection{Post-processor - Computes mean of all} classification scores as overall result and also the standard deviation for anomalies.

4.1.6 Training Database - The CNN model would be trained from the open dataset provided by Skin Cancer MNIST: HAM10000 and SIIM-ISIC Melanoma Classification. Furthermore, each input with high classification score would be added into the training set for better results.

\subsection{Use Case diagram}

Fig 4.2.1 illustrates user activities with the system. The user and the doctor are the two actors interacting with the system. Cloud based mobile application is the main component of the system. There is a direct association between the user and registration, login, images of affected skin area, generate report, report history and email report.

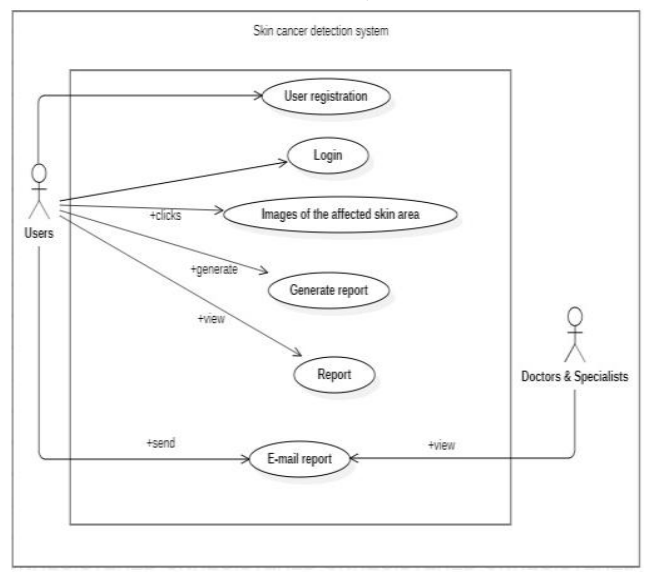

Fig 4.2.1 Use case diagram

The system Use Case is explained as follows:

\section{Cloud-based mobile application system:}

$>$ The users need to register and login when they use the app for the first time.

> The skin image is uploaded to the mobile app for classification and analysis.

> After successfully uploading the file, the user needs to click on "Classify" to perform CNN classification with Inception V3 architecture.

$>$ Following CNN classification, a detailed report would be generated specifying whether the user is suffering from any cardio vascular diseases.

$>$ These reports will be stored in the database as "Past history reports" which can be viewed by the user and could be helpful to the doctor for analysis purpose.

$>$ Furthermore, reports can be sent by users to doctors via email for second opinion.

\section{Design and Implementation}

\subsection{Methodology Flowchart}

Implementation of the system follows the flow specified in Fig 5.1.1.

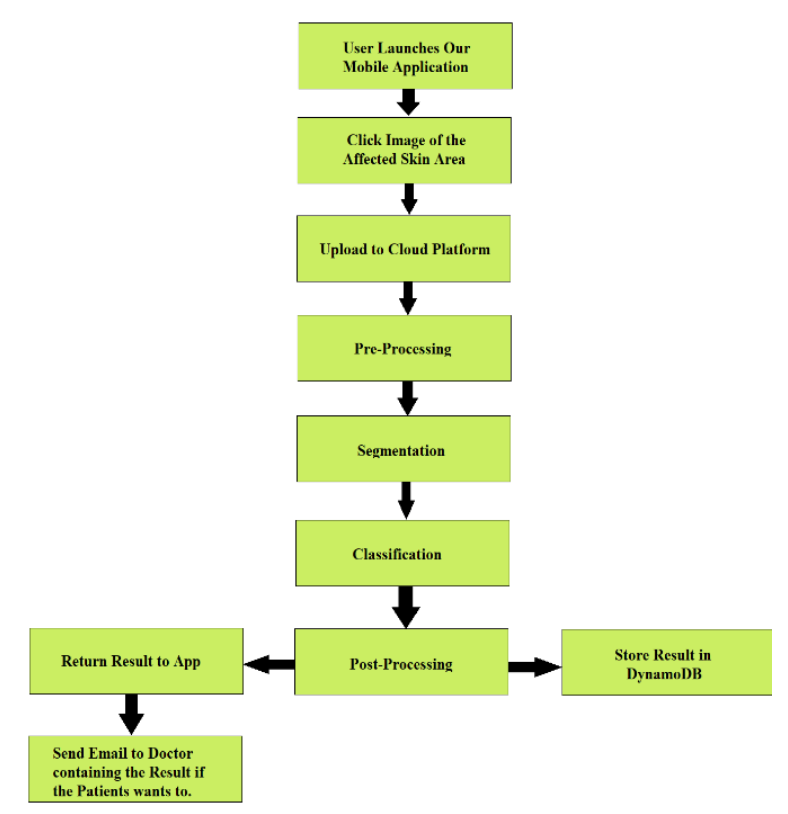

Fig 5.1.1 Methodology Flowchart

User launches the Mobile Application and enters relevant information like the Patient Name, Date of diagnosis etc. 
- The user then clicks the image of the affected skin area via an in-app camera. The image is stored in the mobile's internal storage.

- Upload the image from the app into the backend Cloud-based system for analysis.

- Pre-process the images by standardizing them and performing median filtering.

- Segment the images and perform feature extraction.

- Classify each image into benign or malignant categories using deep CNN.

- Calculate mean of all classification scores and standard deviation to remove any anomaly.

- For the images that have high classification score, store them in a CNN Training database that serves as a growing database for retraining the CNN model for improved accuracy.

- Return overall classification to mobile app.

- Send an email to a doctor containing the test results, if the user wants a second opinion.

\subsection{Image Pre-Processing}

Following steps, as shown in Fig. 5.2.1, will be carried out in this stage in order to make the image compatible with the CNN classifier input:

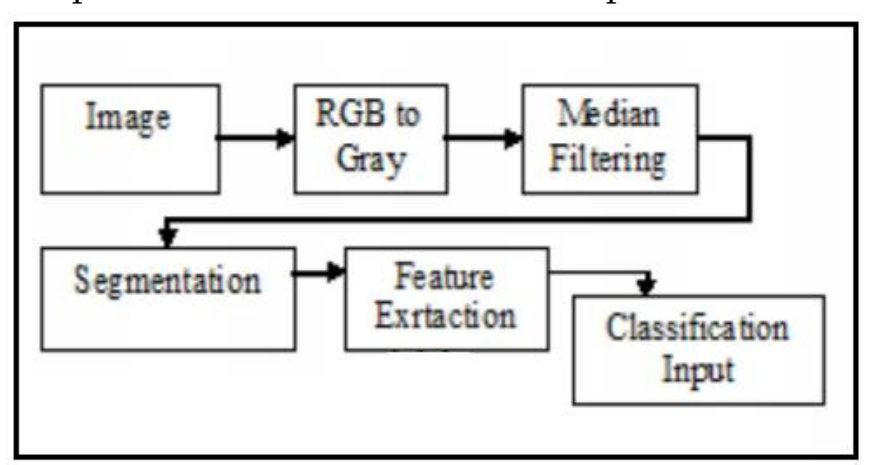

Fig. 5.2.1 Pre-Processing steps

\subsubsection{Standardizing the images:}

The images coming from the mobile application differ in various aspects like the dimension, quality, contrast, file type etc. Thus, it is necessary to have a system that can standardize the images to have universally same attributes before it can be passed on for further processing. So, in this stage a python program would be used to translate all the input images into a form that is universal and easy to work with.

\subsubsection{Median Filtering:}

With the help of median filtering the unwanted part of skin image is removed. After applying median filtering smooth image is obtained. Median filtering is necessary because unwanted portion of an image can reduce the accuracy of classification. The median filter is a non-linear digital filtering technique.

\subsubsection{Segmentation:}

Segmentation is nothing but a partitioning of image so that the region of interest is obtained. Segmentation is applied on smoothed image. Thresh holding technique can be used before segmentation. In this technique, peak value for skin and peak value for lesion will be determined and then threshold will be selected in between these two peak points. The pixel intensity values those are greater than threshold value will be set as 0 while intensity values those are less than threshold value will be set as 1 . The segmented image will look like Fig 5.2.2.

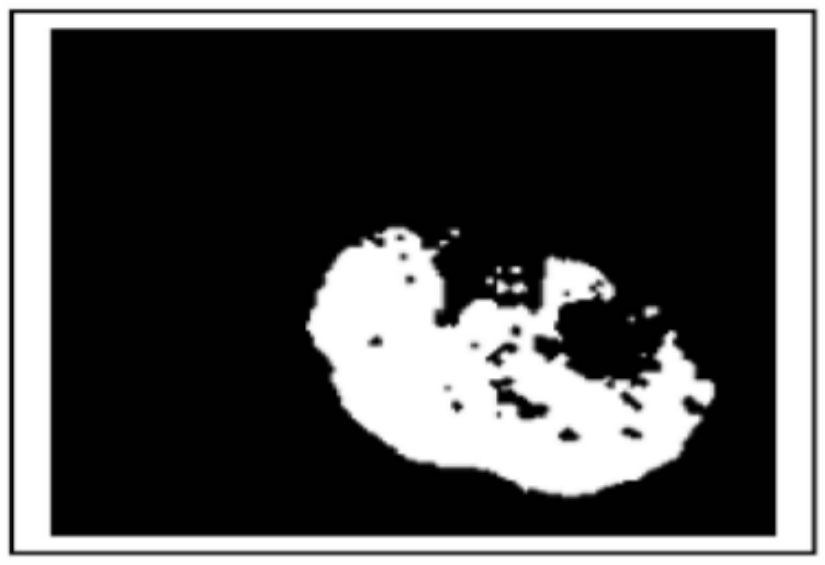

Fig 5.2.2 Segmented image of skin cancer

\subsubsection{Feature Extraction:}

Feature extraction will be done as a part of Convolution Neural Networks but can also be performed separately after segmentation using 
wavelet transform. For Melanoma cancer features resides within the border of lesion. These features are applied on classification algorithm to classify cancer images and skin images. It is essential to extract important features so that the classification results will be improved. Primarily, there are two types of features - color based and texture based.

\subsection{CNN Classifier}

Once the image is pre-processed and standardized, CNNs are naturally the best candidate to identify patterns within the image and classify them into respective classes. Given the variation in color, texture, symmetry and shape of the different classes of tumor, we can use deep neural networks to identify the different classes.

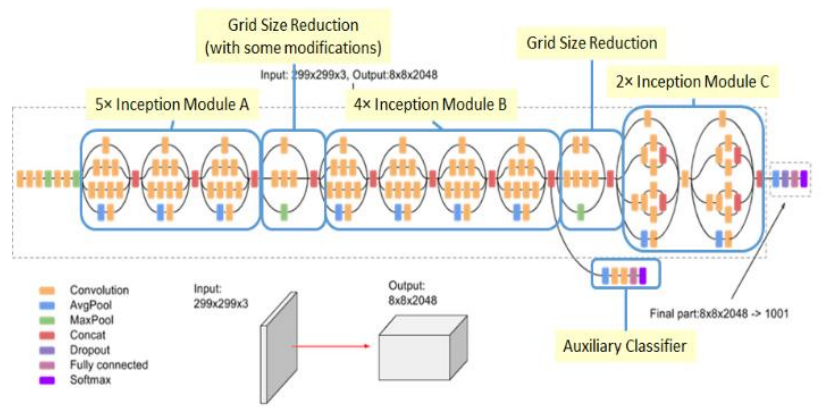

Fig 5.3.1 Inception v3 Architecture

Fig 5.3.1 illustrates Inception v3 architecture which might be used as a CNN Classifier. Inception v3 is 42layer deep learning network that has been shown to attain greater than $78.1 \%$ accuracy on the ImageNet dataset. The model is the culmination of many ideas developed by multiple researchers over the years. It is based on the original paper: "Rethinking the Inception Architecture for Computer Vision" by Szegedy, et. al.

The model itself is made up of symmetric and asymmetric building blocks, including convolutions, average pooling, max pooling, concats, dropouts, and fully connected layers. Batchnorm is used extensively throughout the model and applied to activation inputs. Loss is computed via Softmax.

During the implementation phase of the project, we might also experiment with our own custom CNN architecture model and use whichever model gives us the higher accuracy for the final system.The model itself is made up of symmetric and asymmetric building blocks, including convolutions, average pooling, max pooling, concats, dropouts, and fully connected layers. Batchnorm is used extensively throughout the model and applied to activation inputs. Loss is computed via Softmax.

During the implementation phase of the project, we might also experiment with our own custom CNN architecture model and use whichever model gives us the higher accuracy for the final system.

\section{Experimental Results}

\subsection{Experiment1: Building CNN from Scratch}

We built a model in Pytorch containing 3 convolution layers and 3 max pooling layers placed alternately, followed by fully-connected layer and output layer. Dropout was 30\%.

Intermediate layers uses 'relu' activation function. $\mathrm{O} / \mathrm{P}$ layer uses sigmoid activation function. Optimizer is ' $\mathrm{SGD}$ (Stochastic Gradient Descent)', loss function is 'binary cross entropy'.

Input image is of the size $224 \times 224 \times 3$

After Training the model for 30 epochs on Dataset B, we got a validation accuracy of $84.55 \%$. 


\subsection{Transfer Learning}

Transfer learning involves taking a pre-trained neural network and adapting the neural network to a new, different data set.

\section{Depending on both:}

A.The size of the new data set, and

B.The similarity of the new data set to the original data set

The approach for using transfer learning will be different. We can either use the pre-trained model as a feature extractor or we can fine tune the model based on different cases.

For our Use Case, we are using Inception v3 for transfer learning. Inception v3 has been trained on the large ImageNet Dataset which is quite different to our Skin Cancer dataset, hence we will perform Fine Tuning.

Fine Tuning -

Remove Final Fully Connected layer and add new Fully Connected Layer.

Randomly initialize weights in the fully connected layer.

Initialize the rest of the weights using the pretrained weights.

Retrain entire network.

We used Google's Inception v3 Classifier.

Replaced the final fully connected layers in auxiliary classifier and main classifier as per our requirement.

When we used Adam optimizer, after 20 epochs, we got a validation accuracy of $88.33 \%$

\subsection{Experiment 2A: Transfer Learning - Inception v3 on Dataset A using SGD optimizer.}

When we used Adam optimizer, after 20 epochs, we got a validation accuracy of $82.67 \%$

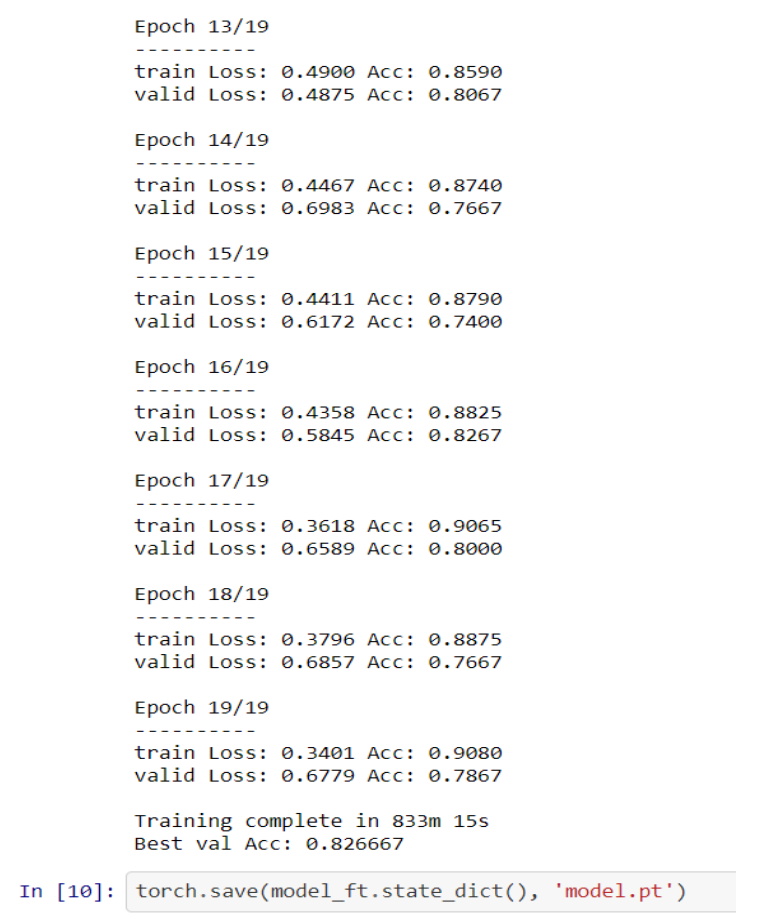

FIG 6.3 Experiment 2A results with Dataset A

\subsubsection{Experiment 2B: Transfer Learning - Inception v3 on Dataset B using Adam optimizer.}

We used Google's Inception v3 Classifier.

Replaced the final fully connected layers in auxiliary classifier and main classifier as per our requirement.

When we used Adam optimizer, after 20 epochs, we got a validation accuracy of $88.33 \%$.

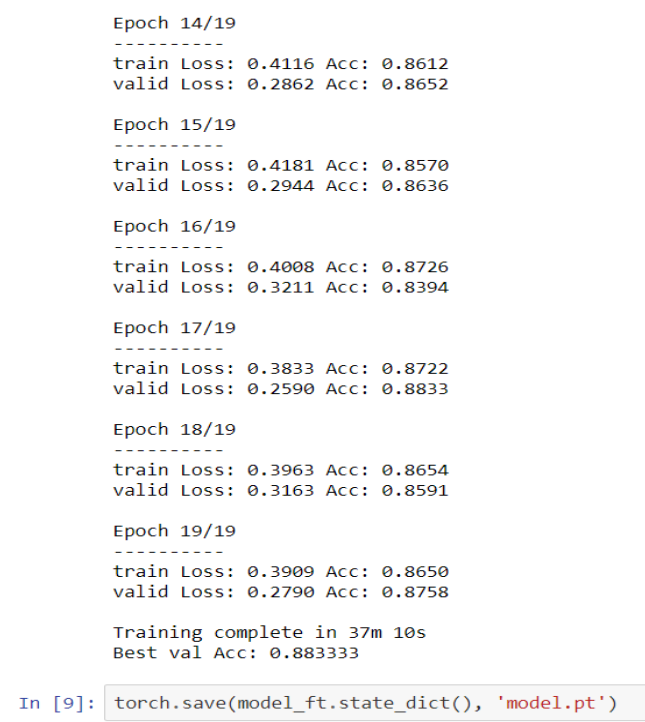

FIG 6.3.1 Experiment 2A results with Dataset B 


\subsection{Experiment 3: Transfer Learning - Inception v3 on Dataset B using SGD optimizer.}

When we used SGD optimizer, after 20 epochs, we got a validation accuracy of $90.75 \%$.

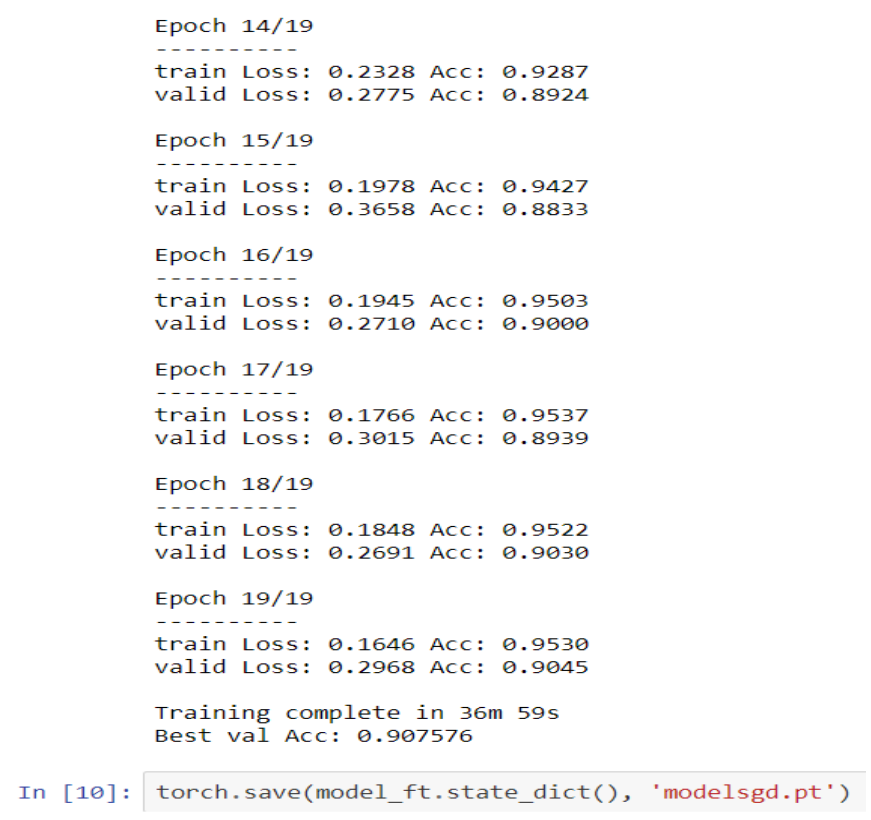

FIG 6.4 Experiment 3 results with Dataset B

\section{TECHNOLOGIES USED}

7.1 Mobile Application: Android Studio (Java)

7.2 Cloud Infrastructure: Amazon Web Services (AWS)

- File Storage: AWS S3

- Serverless Backend: AWS Lambda

- Mobile Application Interfacing: AWS Amplify

- Database: AWS DynamoDB

- Security and User Management: AWS Cognito \& IAM

7.3 Convolution Neural Network: Python

- Training on FloydHub Cloud Platform and AWS Sagemaker.

\subsection{Flask API Server}

- We have created an API Server using Python Flask.

- It listens for GET requests, performs classification and then returns the classification result as json.

- Mobile app uploads photo in S3 Bucket $\rightarrow$ mobile app makes call to Flask API $\rightarrow$ API downloads the most recent photo uploaded to the S3 bucket $\rightarrow$ API performs pre-processing on this photo $\rightarrow$ API performs classification using the trained model $\rightarrow$ API returns result to Mobile App.

\subsection{Mobile UI}
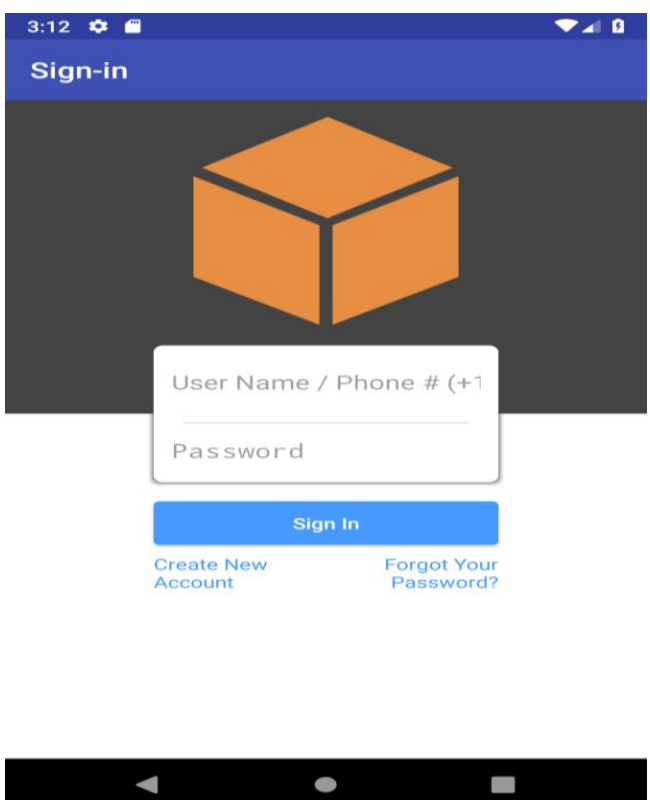

4

\section{口}




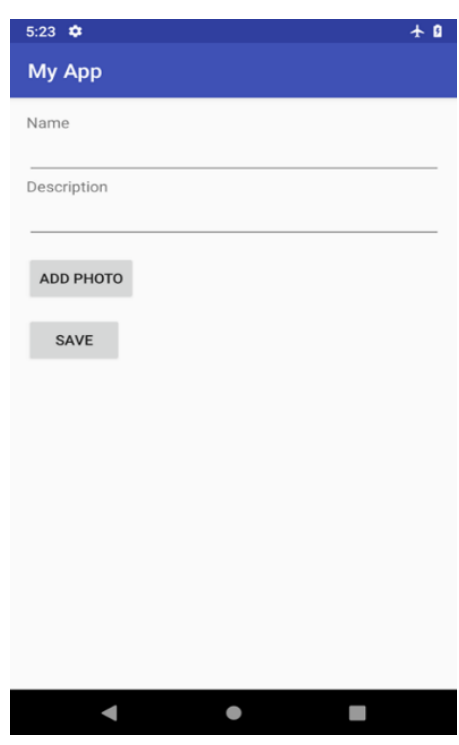

Fig 7.5.1 Mobile UI

\section{Proposed Testing and Result}

\subsection{Proposed Testing}

Multiple levels of experimentation with heart skin cancer images from different sources will be conducted to demonstrate feasibility and robustness of the proposal. Open skin images datasets from Skin Cancer MNIST, SIIM-ISIC (International Skin Imaging Collaboration) will be used to determine accuracy of classification of skin cancer images. These public datasets have a large collection of multisources dermatoscopic images of pigmented lesions with the goal to encourage development of more robust automated skin cancer classification techniques. These datasets will be trained and tested using various methods and the method giving optimal results will be chosen for the implementation of the actual system.

Skin cancer images of affected area will be used for training and testing the CNN model and establish optimal parameters for the result generation and future analysis. The training set will contain $80 \%$ of the image samples from the original dataset and the remaining $20 \%$ of these samples from the dataset will be used as test set. Once the feasibility of the proposal is established with open source dataset, the system will be used to collect skin images in real-life which will be classified using the CNN model trained with the open source datasets.

\section{Proposed Result}
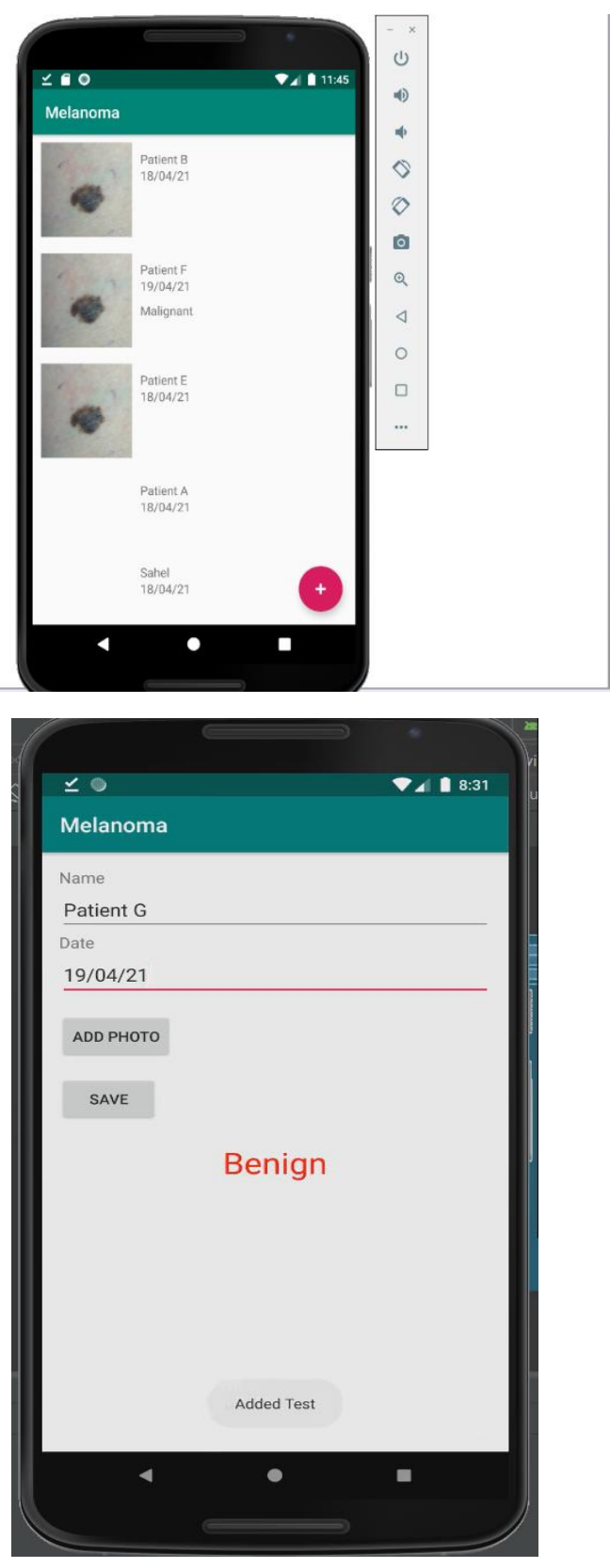

Fig 8.2. Proposed Results 


\section{Performance Analysis}

\begin{tabular}{|c|c|c|}
\hline Study / Research Paper & Method Used & Accuracy / Improvements \\
\hline Our Proposed System & - Transfer Learning (Inception v3) & $\begin{array}{l}\text { - Acc }=90.75 \% \\
\text { - Mobile Solution }\end{array}$ \\
\hline $\begin{array}{l}\text { Nezhadian, F. K., \& Rashidi, S. } \\
\text { (2017). Melanoma skin cancer detection } \\
\text { using color and new texture features. } 2017 \\
\text { Artificial Intelligence and Signal Processing } \\
\text { Conference }\end{array}$ & $\begin{array}{l}\text { - Texture based feature } \\
\text { classification using active } \\
\text { counter model. }\end{array}$ & - $A C C=97 \%$ \\
\hline $\begin{array}{l}\text { Mhaske, H. R., \& Phalke, D. A. } \\
\text { (2013). Melanoma skin cancer detection } \\
\text { and classification based on supervised and } \\
\text { unsupervised learning. } 2013 \text { International } \\
\text { Conference on Circuits, Controls and } \\
\text { Communications }\end{array}$ & $\begin{array}{l}\text { - Classification based on Neural } \\
\text { networks and Support vector } \\
\text { machine (SVM). }\end{array}$ & - $A C C=80-90 \%$ \\
\hline $\begin{array}{l}\text { Dai, X., Spasic, I., Meyer, B., Chapman, S., \& } \\
\text { Andres, F. (2019). Machine Learming on } \\
\text { Mobile: An On-device Inference App for Skin } \\
\text { Cancer Detection. } 2019 \text { Fourth } \\
\text { International Conference on Fog and } \\
\text { Mobile Edge Computing } \\
\text { (FMEC). doi:10.1109/fmec.2019.8795362 }\end{array}$ & $\begin{array}{l}\text { Classification using } \\
\text { convolutional neural Networks } \\
\text { (CNN) and Deep Learning }\end{array}$ & - $A C C=75.2 \%$ \\
\hline
\end{tabular}

Fig. 9 Performance Analysis

\section{CONCLUSION \& FUTURE SCOPE}

In recent days, skin cancer is seen as one of the most Hazardous form of the Cancers found in Humans of which Melanoma is the most unpredictable. We aim to maximize the accuracy of our CNN classification model which will be used to detect Melanoma cancer. Medical imaging as non-invasive tool with high accuracy and performance has been attracted. Therefore, mechanisms for processing image is used to diagnose and treat disease without invasive activities.

The UI for this application is an android app which is very fast and user friendly. User can simply scan any area of skin using this app and the trained CNN model at the back will detect whether its malignant or not. It proves to be a better diagnosis method than the conventional Bioscopy method. The most important step is to segment image with high accuracy. Hence trained CNN model is used and initial district will be determined by user to increase accuracy. Texture -based features and RGB components is used to extract image features.

TC features for approximation matrices of wavelet transform is selected as most efficient feature. It is painless and timeless process than biopsy method. It is even costless checkup that user can have from anywhere which is more advantageous to patients. In today's world, where artificial intelligence is playing an important role in medical fields, the accuracy of this application can be increase with upcoming machine learning models.

With the increase of human population, a lot of infections and skin diseases are occurring in our day to day routine which are not people aware about. This application can be further developed to detect more kind of skin infections and diseases which required more studies and research reports on large and complete database will be needed to increase the performance and speed of diagnosis.

\section{REFERENCES}

[1]. H. R. Mhaske and D. A. Phalke, "Melanoma skin cancer detection and classification based on supervised and unsupervised learning," 2013 International conference on Circuits, Controls and Communications (CCUBE), Bengaluru, 2013, pp. 1-5, doi: 10.1109/CCUBE.2013.6718539.

[2]. F. K. Nezhadian and S. Rashidi, "Melanoma skin cancer detection using color and new texture features," 2017 Artificial Intelligence and Signal Processing Conference (AISP), Shiraz, 2017, pp. 1-5, doi: 10.1109/AISP.2017.8324108.

[3]. Tschandl, Philipp, 2018, "The HAM10000 dataset, a large collection of multi-source dermatoscopic images of common pigmented skin lesions", https://doi.org/10.7910/DVN/DBW86T, Harvard Dataverse

[4]. SIIM-ISIC Melanoma Classification Dataset.

[5]. https://www.frontiersin.org/articles/10.3389/fme d.2020.00233/full

[6]. Buka-https://www.webmd.com/melanoma-skincancer/features/ai-skin- 
Pawan Sonawane et al Int. J. Sci. Res. Comput. Sci. Eng. Inf. Technol, May-June - 2021, 7 (3) : 312-324

cancer\#: :text=Buka\%3A\%20There\%27s\%20one

\%20technology\%20called,serious\%20skin\%20ca

ncers\%2C\%20including\%20melanoma

[7]. https://mc.ai/skin-cancer-image-

classification $\%$ E2\%80\%8A-\%E2\%80\%8Aan-

educational-guide/?am

\section{Cite this article as :}

Pawan Sonawane, Sahel Shardhul, Raju Mendhe, "Cloud based mobile solution for early detection of Skin Cancer using Artificial Intelligence", International Journal of Scientific Research in Computer Science, Engineering and Information Technology (IJSRCSEIT), ISSN : 2456-3307, Volume 7 Issue 3, pp. 312-324, May-June 2021. Available at doi : https://doi.org/10.32628/CSEIT217327

Journal URL : https://ijsrcseit.com/CSEIT217327 